\title{
The Role of Time in Cosmic Expansion
}

\author{
Naser Mostaghel \\ Department of Civil Engineering, University of Toledo, Toledo, USA
}

Email address:

naser.mostaghel@utoledo.edu

\section{To cite this article:}

Naser Mostaghel. The Role of Time in Cosmic Expansion. American Journal of Astronomy and Astrophysics. Vol. 6, No. 1, 2018, pp. 9-20. doi: 10.11648/j.ajaa.20180601.12

Received: January 22, 2018; Accepted: February 5, 2018; Published: March 2, 2018

\begin{abstract}
By treating time as an independent variable free of space-time the role of time in cosmic expansion is clarified. We show that this treatment of time is consistent with General Relativity, and addresses the quandaries of dark or vacuum energy. We consider the current of time to be composed of many time waves. As the current flows, the number of its waves keeps increasing. It is shown that the cumulative sum of the periods of these waves represents the stretching-time, the redshift, $z$, represents the stretching velocity, and the quantity $z^{2} / t$ represents the stretching acceleration of the stretching-time. By isolating time from space-time we find a simple equation which is developed based only on time and its kinematics. The validity of this equation is confirmed first through the conformity of its predictions with Einstein's three predictions, namely the precession of Mercury's orbit, the bending of light by the sun's gravity, and the gravitational time dilation. Second, its validity is further confirmed through its consistency with three different sets of observational data as well as with the recent LIGO/Virgo gravitational waves measurement. It is shown that the flow of stretching-time is propelled by the energy released at the big bang. Further, the Hubble constant is estimated analytically. Also a possible source and the quantity of what is called dark energy are identified. It is concluded that the time model may clear the way to a quantum mechanical description of the cosmos.
\end{abstract}

Keywords: Cosmic Expansion, Dark Energy, Gravitation, Redshift, Time

\section{Introduction}

Ever since Edwin Hubble [1], through observations, showed that the universe is expanding, there have been tremendous research efforts to develop a realistic model of the universe that is consistent with observational measurements. The standard model $\Lambda C D M$ has been the culmination of this effort. This model is in terms of a limited number of parameters fine tuned to represent the ingredients of the universe. And it offers dark energy as the propulsive agent driving the expansion. The model is consistent with observational data, but it does not explain the assumptions of large scale homogeneity and isotropy that lie at the foundation of its formulation. It also does not shed any light on the cosmic acceleration that was discovered in 1998 [2], and has been confirmed by two independent teams $[3,4,5]$. As a remedy, Alan Guth [6, 7, 8], assuming an early inflationary stage, shows how a process of an exponential expansion at the birth of the universe can explain how the universe has grown to be so smooth and uniform. It also can explain the formation of large-scale structures [9]. But the assumption of inflation at the initiation of the universe can also lead to a non-flat universe [10, 11, 12]. Guth's inflationary model offers a false vacuum as the propulsive force driving the expansion.

Considering only time and its kinematics, and considering the current of time to be composed of a number of time waves with different frequencies, it is shown that as time flows, the number of its waves keeps increasing. The cumulative sum of the periods of these time waves represents the stretching-time. The proposed model of time reveals that the redshift, $z$, is the velocity of the stretching-time, and the quantity $z^{2} / t$ is the stretching-time's acceleration.

It is seen that treating time in isolation, free of entanglement with space-time, yields a very simple equation. We use this equation to predict the precession of Mercury's orbit, the bending of light by the sun's gravity, and, through an application for the Geographic Positioning System, the gravitational time dilation. All these predictions are essentially the same as the Einstein general relativity predictions. It will be seen that the time dilation caused by acceleration is the unique cause of all the above three 
phenomena. The time model's validity is further confirmed through the remarkable agreement of its prediction with three different sets of observational data and with the recent observations of gravitational waves [13-17].

In the time model, we represent the cosmological time as a slender elastic rod, a "time-rod," resting between a faraway point source and the earth. Summing up the periods of all the axial frequencies of the free vibration of this time-rod yields the stretching-time, which then is multiplied by its spatialvelocity to yield the spatial-distance between a point source and the earth. This model offers an alternative to the standard and the inflationary models of cosmology. It yields the timehistory of the expansion of the universe from the big bang to the present. We make no assumptions, except those implicit in Wein's displacement law and in the temperature-redshift relation. It will be shown that the time wave propagation is fueled by the energy released at the big bang. Further, this model of time leads us to identify both the source and the quantity of what is called dark or vacuum energy.

Next, in section 2, we first evaluate the frequencies of the free axial vibration of the time-rod and then evaluate the value of the stretching-time between far away point sources and the earth. In section 3, we evaluate the rates at which the stretching-time increases, that is, the velocity and the acceleration of the stretching-time. In section 4, we discuss the time cycles and formulate the acceleration time dilation equation. In section 5, we validate the model through the conformity of its predictions with those of general relativity and its consistency with observational data. In section 6 , we discuss the effect of the energy released at the big bang and its role as the driver of stretching-time. We also make an analytical estimate for the Hubble constant and identify both the source and the quantity of what is called dark energy. Conclusions and remarks are presented in section 7.

\section{Evaluation of Stretching-Time and Frequencies}

Given the age of the universe to be $t_{0}=13.8$ giga years, I want to evaluate the value of stretching-time between a far away point source, such as a galaxy, and the earth. Due to the increasing number of time waves, the value of the stretchingtime has been increasing since the birth of time. We consider this value to be the cumulative sum of a series of periods of time waves. Therefore, to evaluate the stretching-time to the point source, we first need to determine the numbers of time waves and their corresponding wave periods. To this end we represent time as a uniform slender elastic rod, a "time rod", stretching from its mid-point, along the $\mathrm{x}$-axis as shown in Figure 1, between the point source and the earth. Given the age of the universe, $t_{0}$, we first find the frequencies of the axial free vibration of the time rod of length $L / 2$ stretched from point $\mathrm{O}$ to the earth. Then by summing up all the periods of the time waves corresponding to the axial frequencies of this time rod, we find the value of the stretching-time, $T_{s}$, between the point $\mathrm{O}$ and the earth. Also because time flows forward both toward the earth and toward the point source simultaneously, the same temporal time, $t$, is the elapsed time between the earth and the point source. Consequently, as can be inferred from Figure 1, the spatial distance between the earth and the point source is, numerically, twice the distance between the mid-point $\mathrm{O}$ and the earth.

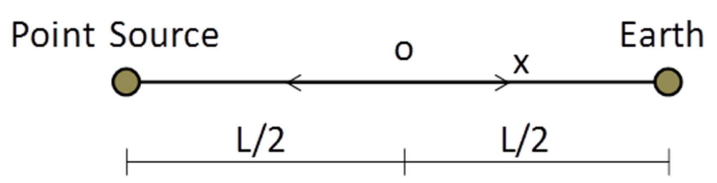

Figure 1. A Representation of the Elastic Time Rod.

The axial wave propagation along a slender elastic rod, with no external forces present, is described by the partial differential equation

$$
u^{\prime \prime}(x, t)-\frac{1}{c^{2}} \ddot{u}(x, t)=0
$$

where prime represents differentiation with respect to $\mathrm{x}$, and dot represents differentiation with respect to time $t$, and the constant, $c$, represents the speed of propagation of the time waves, which is considered to be the same as the speed of light. Let $L$ denote the length of the elastic rod. The initial conditions are:

$$
\begin{gathered}
u(x, 0)=f(x) \\
\dot{u}(x, 0)=0
\end{gathered}
$$

and the boundary conditions are:

$$
\begin{aligned}
u(0, t) & =0 \\
u^{\prime}\left(\frac{L}{2}, t\right) & =0
\end{aligned}
$$

Here our main interest is the values of the axial wave propagation frequencies and periods. The solution of equation (1), subject to the specified boundary conditions, yields the frequencies $[18,19]$ as

$$
\omega_{n}=(2 n-1) \frac{\pi c}{L} \rightarrow f_{n}=\frac{\omega_{n}}{2 \pi}=(2 n-1) \frac{c}{2 L}
$$

Therefore the periods are given by

$$
p_{n}=\frac{1}{f_{n}}=\frac{2}{2 n-1} \frac{L}{c}, \mathrm{n}=1,2,3, \ldots
$$

Replacing the length $L$ by $c t_{0}$, where $t_{0}$ represents the cosmological age of the universe, one obtains

$$
p_{n}=\frac{2}{2 n-1} t_{0}
$$

The period $p_{n}$ represents the temporal length of the nth time wave. Thus the spatial length of the nth time wave is given by

$$
\lambda_{n}=c p_{n}=\frac{2}{2 n-1} c t_{0}
$$

It should be noted that since the speed of light, c, is constant, any change in the lengths of the waves is due to the 
change in the period of the time waves. If the lengths of a time wave at the source and at the location of observation are denoted by $\lambda_{e}$ and $\lambda_{o}$ respectively, then using the above relations one obtains

$$
\begin{aligned}
& n_{e}=\frac{1}{\lambda_{e}} c t_{0}+\frac{1}{2} \\
& n_{o}=\frac{1}{\lambda_{o}} c t_{0}+\frac{1}{2}
\end{aligned}
$$

But by definition of the cosmological redshift we have

$$
\frac{\lambda_{o}}{\lambda_{e}}=1+\mathrm{z}
$$

where $z$ represents the cosmological redshift. Substitution of the above relation back into equation (11) yields

$$
n_{o}=\frac{1}{\lambda_{e}(\mathrm{z}+1)} c t_{0}+\frac{1}{2}
$$

In order to evaluate the numbers $n_{e}$ and $n_{o}$ we first determine, as an example, the value of the redshift, $z$, and the length of the time wave, $\lambda_{e}$, for a point source at the horizon. To this end we use the fact that the temperature from the $\mathrm{CMB}$ radiations at the time of decoupling was about $\tau_{\text {then }}=$ $3000^{\circ} \mathrm{K}$, and the black body radiation temperature now is $\tau_{\text {now }}=2.72548^{\circ} \mathrm{K}$ [20]. From the following relations for the cosmological redshift

$$
\begin{gathered}
\frac{\lambda_{o}}{\lambda_{e}}=\frac{\tau_{\text {then }}}{\tau_{\text {now }}}=\frac{3000^{\circ} \mathrm{K}}{2.72548^{\circ} \mathrm{K}}=z+1 \\
\tau_{\text {then }}=\tau_{\text {now }}(z+1)=2.72548(z+1)
\end{gathered}
$$

where $\lambda_{o}$ and $\lambda_{e}$ are the observed and emitted wave lengths respectively, we evaluate $\lambda_{e}$ through Wien's displacement law [21, 22], which yields

$$
\lambda_{e}=\frac{2.8977729 \times 10^{-3} \mathrm{~K} \mathrm{~m}}{\tau_{\text {then }}}=\frac{2.8977729 \times 10^{-3} \mathrm{~K} \mathrm{~m}}{2.72548(z+1)}=\frac{1.0632156 \times 10^{-3}}{(z+1)}
$$

Now substituting the above value for $\lambda_{e}$ back into equation (10) we obtain

$$
\mathrm{n}_{e}=\frac{(z+1) c t_{0}}{1.0632156 \times 10^{-3}}+\frac{1}{2}
$$

Each period is defined by equation (8). Thus the number of periods to be summed up is given by

$$
\Delta \mathrm{n}=n_{e}-n_{o}=\frac{c t_{0} \mathrm{z}}{\lambda_{e}(\mathrm{z}+1)}=\frac{c t_{0} \mathrm{z}}{1.0632156 \times 10^{-3}}
$$

As this is a very large number, to sum up the periods, we divide the above number to a number of segments and sum up the segments. To simplify, we represents the number of segments by $13.8 \mathrm{~m}$. To this end, using equations (17) and (18), we represent the segment $\Delta \sigma_{i}$ as

$$
\begin{aligned}
\Delta \sigma_{i}= & n_{e}-\frac{i}{13.8 m} \Delta \mathrm{n}=\left[\frac{(z+1) c t_{0}}{1.0632156 \times 10^{-3}}+\frac{1}{2}\right]- \\
& \frac{i}{13.8 m} \frac{c t_{0} \mathrm{z}}{1.0632156 \times 10^{-3}}
\end{aligned}
$$

Thus, using equation (8), the sum of the periods of the stretching-time is given by

$$
\begin{gathered}
\mathrm{T}_{\mathrm{s}}\left(t_{i}\right)=\sum_{n=\Delta \sigma_{i}}^{\mathrm{ne}} p_{n}=\sum_{n=\Delta \sigma_{i}}^{\text {ne }} \frac{2}{(2 n-1)} t_{o}, \\
0 \leq i \leq m
\end{gathered}
$$

According to the above relation, the stretching-time is the sum of discrete periods. Clearly as $t_{i}$ grows from its lowest value to its present-time value, $t_{0}$, the stretching-time increases due to the increase in the numbers of periods of the time waves in the summation. Further the summation of the discrete periods in equation (20) indicates that the emergence of the stretching-time is a discrete process.

To exploit the model, we first evaluate the value of the stretching-time between the earth and the horizon (the boundary of the observable universe or the decoupling time). For $\tau_{\text {Horizon }}=3000^{\circ} \mathrm{K}$, equation (15) yields $z=1099.72$. This value for the redshift is consistent with the $z=1090$ proposed by the WMAP team [23]. The age of the horizon is less than the age of the universe but, as an example, we consider it to be the same. Thus considering $t_{0}=13.8 \mathrm{~Gy}$, substituting the above value for $\mathrm{z}$ in equations (17) and (19), then equation (20) yields the equation for the time-history of the stretching-time to a point source at the horizon. A plot of this time-history is presented in Figure 2. We have chosen the number $m$ to be 100 . Increasing this number to numbers larger than 100, except for yielding a smoother curve, makes the computation much more time consuming. Our purpose is to show the trend schematically.

Equation (20) can also be used to evaluate the value of the stretching-time to any far away point sources. For example we consider the four latest spectroscopically confirmed galaxies, whose measured redshifts are summarized in Table 1. Substituting each galaxy's redshift into equations (17) and (19), then equation (20) yields the corresponding value of stretching-time at the present time. These calculated stretching-times are also presented in Table 1 in bold. To find the stretching-time for a time beyond the present time, one just substitutes the corresponding age of the universe in equation (20). Except for the expanded scales, the timehistory would look the same as in Figure 2.

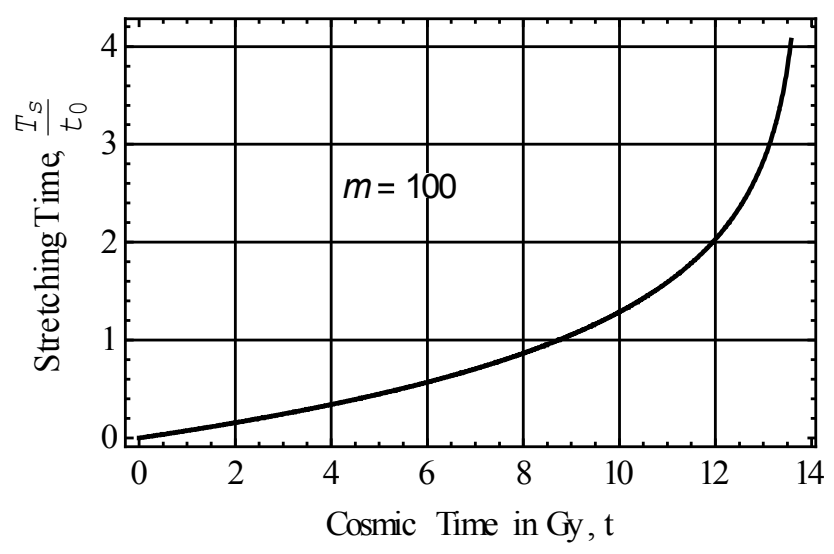

Figure 2. Schematic Time-History for StretchingTime to Horizon. 
Table 1. Horizon and Galaxies, Calculated Present-Time Values.

\begin{tabular}{llll}
\hline Name & Ref. & Redshift $\mathbf{z}$ & $\frac{\mathbf{T}_{\mathbf{s}}\left(\boldsymbol{t}_{\mathbf{0}}\right)}{\boldsymbol{t}_{\mathbf{0}}}$ \\
\hline Horizon & {$[23]$} & 1099.72 & 7.00372 \\
GN-z11 & {$[24]$} & 11.09 & 2.49238 \\
EGSY8p7 & {$[25]$} & 8.683 & 2.27037 \\
EGS-zS8-1 & {$[26]$} & 7.7302 & 2.16679 \\
z8GND 5296 & {$[27]$} & 7.51 & 2.14124 \\
\hline
\end{tabular}

\section{The Stretching-Time Velocity and Acceleration}

The slope of the curve in Figure 2 is the rate at which the sum of the periods of the stretching-time waves is changing. Therefore this slope represents the temporal velocity of the stretching-time. In this section, first we show that, at each instant of time, the numerical value of this slope is the same as the cosmological redsift, $z$. The stretching-time $T_{s}\left(t_{i}\right)$ in equation (20) represents the sum of the periods of the time waves up to the time $t_{i}$. The rate of change of $\mathrm{T}_{\mathrm{s}}\left(t_{i}\right)$ at time $t_{i}$ is given by

$$
\frac{\mathrm{d} T_{S}\left(t_{i}\right)}{\mathrm{dt}}=\frac{\left[T_{S}\left(t_{i}\right)-T_{S}\left(t_{i-1}\right)\right]}{t_{i}-t_{i-1}}
$$

where $\frac{\mathrm{d} T_{S}\left(t_{i}\right)}{\mathrm{dt}}$ represents the stretching-time's temporal velocity, and the differentiation is with respect to $t_{i}=\frac{i}{m} t_{0}$. To see the trend, as an example, we again set the number $\mathrm{m}=100$ in equation (19). Substituting for $T_{S}\left(t_{i}\right)$ from equation (20) into the above equation yields the temporal velocity. Using equation (21), a schematic time-history for the stretching-time's temporal velocity for a point source at the horizon is evaluated and presented in Figure 3.

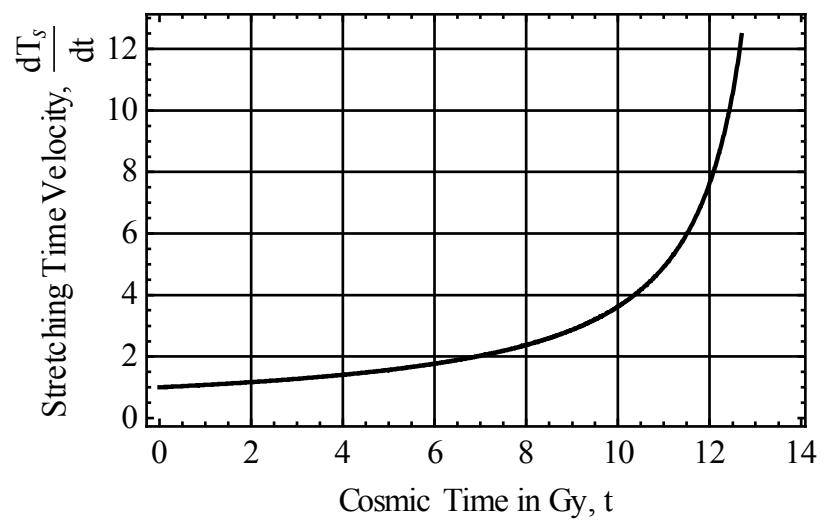

Figure 3. Schematic Time-History of Stretching-Time's Velocity.

The present-time value of time's temporal velocity, $\frac{\mathrm{d} T_{S}\left(t_{0}\right)}{\mathrm{dt}}$, is very sensitive to the selected value for the number, $m$. But as reported in Table 2, this sensitivity diminishes with increase in the number $\mathrm{m}$. And in each case the temporal velocity of time converges to the corresponding value of the redshift. Thus it is concluded that physically, cosmological redshift is the same as the present-time value of the rate of change of stretching-time, which is the same as the temporal velocity of stretching-time, that is

$$
\frac{\mathrm{d} T_{S}\left(t_{0}\right)}{\mathrm{dt}}=Z
$$

The temporal acceleration of the stretching-time is evaluated through the following equation

$$
\frac{\mathrm{d}^{2} T_{S}\left(t_{i}\right)}{\mathrm{dt}^{2}}=\frac{\left[\frac{\mathrm{d} T_{S}\left(t_{i}\right)}{\mathrm{dt}}-\frac{\mathrm{d} T_{S}\left(t_{i-1}\right)}{\mathrm{dt}}\right]}{t_{i}-t_{i-1}}
$$

where $\frac{\mathrm{d} T_{S}\left(t_{i}\right)}{\mathrm{dt}}$ is defined by equation (21). Again to see the trend, as an example, we set $m=100$. Using the above equation, the time-history for time's temporal acceleration for a point source at the horizon is evaluated and presented in Figure 4. As in the case of temporal velocity, the present-time value of the stretching-time temporal acceleration is also very sensitive to the segment number, $m$. As shown in Table 2 , as the number $\mathrm{m}$ is increased to between $10^{4}$ and $10^{6}$, the present-time values of the temporal accelerations, for all redshifts, converge and we discover that

$$
\begin{gathered}
\frac{\mathrm{d}^{2} T_{s}\left(t_{0}\right)}{\mathrm{dt}^{2}}=\frac{\mathrm{z}^{2}}{t_{0}}=a_{t} \rightarrow \\
\frac{d^{2} R\left(t_{0}\right)}{d t^{2}}=\mathrm{c} \frac{\mathrm{d}^{2} T_{s}\left(t_{0}\right)}{\mathrm{dt}^{2}}=\mathrm{c} a_{t}=\mathrm{c} \frac{\mathrm{z}^{2}}{t_{0}}
\end{gathered}
$$

where $t_{0}$ is the present-time age of the universe, $a_{t}$ is the rate at which the stretching-time, $T_{s}\left(t_{0}\right)$, is accelerating, and $\frac{d^{2} R\left(t_{0}\right)}{d t^{2}}$ represents the spatial acceleration.

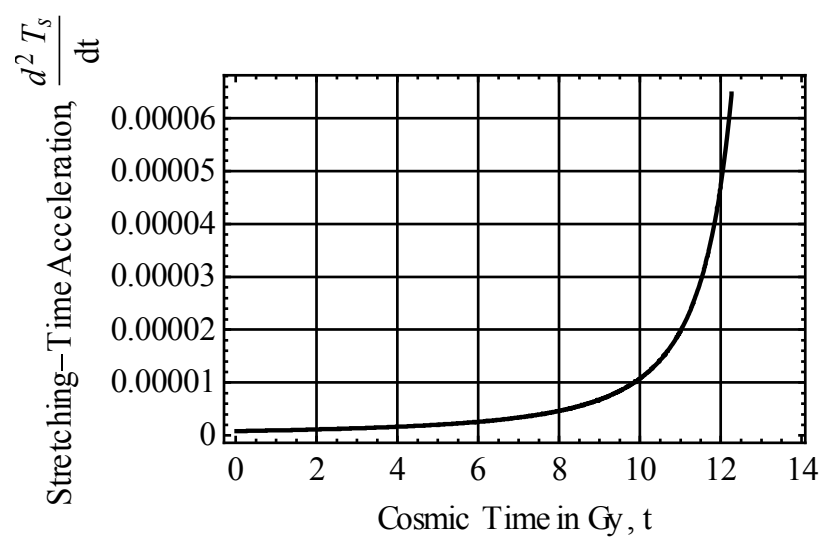

Figure 4. Schematic Time-History of Stretching-Time Acceleration.

\section{Acceleration Time Dilation and Time Cycles}

Based on equation (24)

$$
\mathrm{z}^{2}=a_{t} t_{0}
$$

Now the change in the value of $z^{2}$ between two locations along the current of time is given by

$$
\Delta \mathrm{z}^{2}=z_{2}^{2}-z_{1}^{2}=a_{t 2} t_{02}-a_{t 1} t_{01}
$$

In the following it will be shown that $\Delta \mathrm{z}^{2}$ represents an acceleration time dilation. According to equation (24), $a_{t}$ represents the temporal acceleration of the stretching-time. 
This temporal acceleration is related to the spatial acceleration through the speed of light as

$$
a=c a_{t}=\frac{\mathrm{cz}^{2}}{t_{0}} \rightarrow \mathrm{z}^{2}=a \frac{t_{0}}{c}
$$

Therefore the change in $\mathrm{z}^{2}$ can be represented by

$$
\Delta \mathrm{z}^{2}=z_{2}^{2}-z_{1}^{2}=a_{2} \frac{t_{02}}{c}-a_{1} \frac{t_{01}}{c}
$$

Considering the spatial accelerations to be the same as the gravitational acceleration (Equivalence Principle) and using Newton's law of gravitation together with equation (27) one obtains

$$
\mathrm{z}^{2}=a \frac{t_{0}}{c}=\frac{G M}{\left[R\left(t_{0}\right)\right]^{2}} \frac{t_{0}}{c}
$$

Therefore, using the above relation, substitutions into equation (28) yield

$$
\Delta \mathrm{z}^{2}=z_{2}^{2}-z_{1}^{2}=\frac{G M}{\left[R ( t _ { 0 2 } ) \left[^{2}\right.\right.} \frac{t_{02}}{c}-\frac{G M}{\left[R ( t _ { 0 1 } ) \left[^{2}\right.\right.} \frac{t_{01}}{c}
$$

Now substituting $c t_{01}$ for $R\left(t_{01}\right)$ and $c t_{02}$ for $R\left(t_{02}\right)$ in the above relation one obtains

$$
\Delta \mathrm{z}^{2}=z_{2}^{2}-z_{1}^{2}=\frac{G M}{c^{3} t_{02}}-\frac{G M}{c^{3} t_{01}}
$$

The above equation reflects the fact that $\Delta \mathrm{z}^{2}$ represents the change in the present time along the current of time. This change in time is the time-dilation due to gravitational acceleration, and it is given by

$$
\delta t=\Delta \mathrm{z}^{2}=z_{2}^{2}-z_{1}^{2}=\frac{G M}{c^{3} t_{02}}-\frac{G M}{c^{3} t_{01}}
$$

\begin{tabular}{|c|c|c|c|c|c|}
\hline \multicolumn{2}{|c|}{ Source } & $m 10^{2}$ & $m 10^{4}$ & $m 10^{6}$ & $m 10^{8}$ \\
\hline \multirow{5}{*}{$\begin{array}{l}\text { Velocity } \\
\dot{T}_{s}\left(t_{0}\right)=z\end{array}$} & Horizon & 2199.81 & 1104.13 & 1099.77 & 1099.72 \\
\hline & GN-z11 & 11.1348 & 11.0904 & 11.090 & \\
\hline & EGSY8p7 & 8.71043 & 8.68327 & 8.6830 & \\
\hline & EGS-zs8-1 & 7.75193 & 7.73042 & 7.7302 & \\
\hline & z8GND 5296 & 7.53051 & 7.51020 & 7.5100 & \\
\hline \multirow{3}{*}{$\begin{array}{l}\text { Acceleration } \\
\ddot{T}_{s}\left(t_{0}\right)\end{array}$} & Horizon & 0.34486 & 0.98428 & 0.99988 & \\
\hline & GN-z11 & 0.98414 & 0.99984 & & \\
\hline & EGSY8p7 & 0.98755 & 0.99981 & & \\
\hline \multirow[t]{2}{*}{$\overline{\mathrm{z}^{2} / t_{0}}$} & EGS-zs8-1 & 0.98891 & 0.99989 & & \\
\hline & z8GND 5296 & 0.98922 & 0.99989 & & \\
\hline
\end{tabular}

Alternatively this time dilation is given by

$$
\delta t=z_{2}^{2}-z_{1}^{2}=\frac{G M}{c^{2} R\left(t_{02}\right)}-\frac{G M}{c^{2} R\left(t_{01}\right)}
$$

Table 2. Sensitivity of the Stretching-Time Rates to the Segment Number, $m$.
Based on equations (15), (20), (22), and (24) I have calculated numerical values for the stretching-time and its velocity and acceleration for temperatures varying from $3000^{\circ} \mathrm{K}$, at the horizon, to the Plank temperature of $1.41686 \times 10^{32} \mathrm{~K}$ at the big bang. The redshift corresponding to each temperature is the redshift at the horizon raised to the power $n$. As listed in Table $3, n$ varies from 1 to 10.4285 , which is its value at the Plank temperature. All the time values in Table 3 are in Gy. It is clear that for redshifts larger than $1099.72^{n}$, that is for $n \geq$ 1 , the last four columns in Table 3 are all repeating themselves. This is due to the centrifugal character of the trajectory of time as implied by equations (22) and (24). Based on the repeated values in Table 3 , the nondimensioned stretching-time can be represented by

$$
\frac{\mathrm{T}_{\mathrm{s}}}{\mathrm{t}_{0}}=\psi_{\mathrm{n}}+\delta \psi_{\mathrm{n}}
$$

But according to the penultimate column of Table 3

$$
\delta \psi_{1}=\delta \psi_{2}=\delta \psi_{3}=\ldots=\delta \psi_{\mathrm{n}}=\delta \psi=0.720
$$

Considering the repeated values of the term $\frac{T_{S}}{n t_{0}}$ in the table, it is concluded that the trajectory of time is composed of circles. The normalized angular length of each circle, at the present time, is given by

$$
\frac{T_{S}}{n t_{0}}=\frac{\mathrm{T}_{\mathrm{S}}}{\mathrm{t}_{0}}=2 \pi+\delta \psi=2 \pi+0.720
$$

For calculating the time distance of galaxies from the earth, it should be noted that the maximum time distance along the trajectory of time would be half a cycle. Thus in such a case the value of $\delta \psi$ has to be reduced by the factor of $1 / 2$. Therefore the time dilation in each half circle of time is given by

$$
\delta\left(\mathrm{t}_{0}\right)=(\delta z)^{2}=\frac{1}{2}\left(\frac{\mathrm{T}_{\mathrm{s}}}{\mathrm{t}_{0}}-2 \pi\right)=\frac{0.720}{2}
$$

\begin{tabular}{|c|c|c|c|c|c|c|c|}
\hline $\begin{array}{l}\text { Temperature } \\
\text { Kelvin }\end{array}$ & $z=\dot{T}_{s}=\frac{\dot{R}}{C}$ & $z^{2}=\ddot{T}_{s} t_{0}=\frac{\ddot{R}}{c / t_{0}}$ & $n$ & $T_{s}$ & $\frac{T_{s}}{t_{0}}$ & $\delta \psi$ & $\delta z$ \\
\hline 2.72548 & 0 & 0 & $\ldots$ & 0 & 0 & $\ldots$ & $\ldots$ \\
\hline 5 & 0.83454 & 0.69646 & $\ldots$ & 12.2787 & 0.88976 & $\ldots$ & $\ldots$ \\
\hline 10 & 2.66908 & 7.12398 & $\ldots$ & 20.0293 & 1.45140 & $\ldots$ & $\ldots$ \\
\hline 100 & 35.6908 & $1.27383 \times 10^{3}$ & $\ldots$ & 499387 & 3.61875 & $\ldots$ & $\ldots$ \\
\hline 1000 & 365.908 & $1.33889 \times 10^{5}$ & $\ldots$ & 81.5131 & 5.90674 & $\ldots$ & $\ldots$ \\
\hline
\end{tabular}

Consequently the additional change in the redshift due to this time dilation is

$$
\delta z=\sqrt{\frac{0.720}{2}}=0.600
$$

Due to this time dilation effect, in section 5.4, we will replace the term $z$ in equations (17), (18) and (19) by $(z+\delta z)$.

Table 3. Present-Time Status of the Kinematics of Stretching Time. 


\begin{tabular}{|c|c|c|c|c|c|c|c|}
\hline $\begin{array}{l}\text { Temperature } \\
\text { Kelvin }\end{array}$ & $\mathrm{z}=\dot{T}_{s}=\frac{\dot{R}}{c}$ & $z^{2}=\ddot{T}_{s} t_{0}=\frac{\ddot{R}}{c / t_{0}}$ & $n$ & $\frac{T_{s}}{n}$ & $\frac{T_{s}}{n t_{0}}$ & $\delta \psi$ & $\delta z$ \\
\hline 3000 & 1099.723542 & $1.2093910^{6}$ & 1 & 96.6514 & 7.00372 & 0.720 & 0.600 \\
\hline $3.29618 \times 10^{6}$ & $1.20939 \times 10^{6}$ & $1.46263 \times 10^{12}$ & 2 & 96.6388 & 7.00281 & 0.720 & 0.600 \\
\hline $3.62488 \times 10^{9}$ & $1.32999 \times 10^{9}$ & $1.76889 \times 10^{18}$ & 3 & 96.6388 & 7.00281 & 0.720 & 0.600 \\
\hline $3.98637 \times 10^{12}$ & $1.46263 \times 10^{12}$ & $2.13928 \times 10^{24}$ & 4 & 96.6388 & 7.00281 & 0.720 & 0.600 \\
\hline $4.38389 \times 10^{15}$ & $1.60849 \times 10^{15}$ & $2.58723 \times 10^{30}$ & 5 & 96.6388 & 7.00281 & 0.720 & 0.600 \\
\hline $4.82108 \times 10^{18}$ & $1.76889 \times 10^{18}$ & $3.12898 \times 10^{36}$ & 6 & 96.6388 & 7.00281 & 0.720 & 0.600 \\
\hline $5.30185 \times 10^{21}$ & $1.94529 \times 10^{21}$ & $3.78416 \times 10^{42}$ & 7 & 96.6388 & 7.00281 & 0.720 & 0.600 \\
\hline $5.83057 \times 10^{24}$ & $2.13928 \times 10^{24}$ & $4.57653 \times 10^{48}$ & 8 & 96.6388 & 7.00281 & 0.720 & 0.600 \\
\hline $6.41202 \times 10^{27}$ & $2.35262 \times 10^{27}$ & $5.53482 \times 10^{54}$ & 9 & 96.6388 & 7.00281 & 0.720 & 0.600 \\
\hline $7.05145 \times 10^{30}$ & $2.58723 \times 10^{30}$ & $6.69376 \times 10^{60}$ & 10 & 96.6388 & 7.00281 & 0.720 & 0.600 \\
\hline $1.41686 \times 10^{32}$ & $5.19855 \times 10^{31}$ & $2.70249 \times 10^{63}$ & 10.4285 & 96.6388 & 7.00281 & 0.720 & 0.600 \\
\hline
\end{tabular}

\section{Validation of the Proposed Model}

We validate the time model by first showing that its predictions are consistent with the Einstein three tests: namely, the Precession of Perihelion of Mercury, Deflection of Light by the Sun, and Gravitational Redshift. The sole underlying cause in all these three cases, it will be seen, is the time dilation due to acceleration of stretching-time, as predicted by equation (33). And in each case we will see that the results, as calculated using this equation, are essentially the same as the ones that have been predicted by General Relativity. Next we will apply the time model to cosmic expansion and show that the results are remarkably consistent with the observational data. We will further validate the time model by using it to calculate the distances to the sources of the recently observed gravitational waves due to the merging of neutron stars [13-17].

\subsection{Precession of Perihelion of Mercury}

For Mercury we will use the following data:

Speed of light $=2.99792458 \times 10^{8} \mathrm{~m} / \mathrm{s}$,

Gravitational Constant $=G$

$=6.67408 \times 10^{-11} \mathrm{~m}^{3} \mathrm{~kg}^{-1} \mathrm{~s}^{-2}$,

Sun's Mass $=M_{s}=1.98855 \times 10^{30} \mathrm{~kg}$,

Sun's Radius $=R=6.95700 \times 10^{8} \mathrm{~m}$,

Perihelion $=r_{p}=46.001200 \times 10^{9} \mathrm{~m}$,

Aphelion $=r_{a}=69.816 \times 10^{9} \mathrm{~m}$,

Eccentricity $=e=0.205630$,

The major and minor axes of the elliptical orbit of Mercury are given respectively by

$$
\begin{gathered}
a=\frac{\mathrm{r}_{\mathrm{p}}+\mathrm{r}_{a}}{2} \\
b=\sqrt{\mathrm{r}_{\mathrm{p}} \mathrm{r}_{a}}=a \sqrt{1-e^{2}}
\end{gathered}
$$

where $e$ is the eccentricity of Mercury's elliptical orbit. Based on equation (33) the total time dilation per orbital cycle for Mercury at the perihelion, then at aphelion and back to perihelion on its orbit is given by

$$
\delta t=2\left(\mathrm{z}_{p}^{2}-\left(-\mathrm{z}_{a}^{2}\right)\right)=2\left(\frac{G M_{S}}{c^{2} r_{p}}-\left(-\frac{G M_{S}}{c^{2} r_{a}}\right)\right)
$$

where the factor 2 in the above equation accounts for the fact that in each orbital cycle, the major axis is traversed by time twice. Since the perihelion and aphelion are at the opposite sides of the origin, which is the sun's center, a negative sign is assigned to the term $\mathrm{z}_{a}^{2}$. Thus the total time dilation per orbital cycle is given by

$$
\delta t=2\left(\mathrm{z}_{p}^{2}+\mathrm{z}_{a}^{2}\right)=2\left(\frac{G M_{S}}{c^{2} r_{p}}+\frac{G M_{S}}{c^{2} r_{a}}\right)
$$

Substitutions for $r_{p}$ and $r_{a}$ from equations (39) and (40) back into the above equation yield

$$
\delta t=\frac{4 G M_{S}}{c^{2}} \frac{1}{a\left(1-e^{2}\right)}
$$

The Period of Mercury is given by

$$
\mathrm{T}=\left(\frac{4 \pi^{2} a^{3}}{\mathrm{G} M_{S}}\right)^{1 / 2}
$$

Therefore the total time for perihelion advance around the sun per Mercury's orbital period is given by

$$
\Delta \mathrm{T}=\delta t T=\left(\frac{4 G M_{S}}{c^{2}} \frac{1}{a\left(1-e^{2}\right)}\right)\left(\frac{4 \pi^{2} a^{3}}{\mathrm{G} M_{S}}\right)^{1 / 2}
$$

Based on Kepler's laws of planetary motion [28], it can be shown that

$$
\mathrm{h}^{2}=\mathrm{G} M_{s} \mathrm{r}_{a}(1-\mathrm{e})=\left(\mathrm{r}_{a}^{2} \dot{\phi}\right)^{2}
$$

where $h$ is a constant and $\dot{\phi}$ is the angular velocity at the apogee of Mercury's orbit. But as shown in Figure (5), considering that the perihelion advance around the sun is circular, the eccentricity e $=0$. Thus

$$
\dot{\phi}=\sqrt{\frac{\mathrm{G} M_{S}}{\mathrm{r}_{a}^{3}}}
$$

For the circular precession, $\dot{\phi}$ must be a constant. 
Therefore the perihelion precession per cycle of Mercury's orbit is given by

$$
\begin{gathered}
\Delta \phi=\dot{\phi} \Delta \mathrm{T}=\sqrt{\frac{\mathrm{G} M_{S}}{\mathrm{r}_{a}^{3}}}\left(\frac{4 G M_{S}}{c^{2}} \frac{1}{a\left(1-e^{2}\right)}\right)\left(\frac{4 \pi^{2} a^{3}}{\mathrm{G} M_{S}}\right)^{1 / 2}= \\
\frac{8 \pi G M_{S}}{c^{2} a\left(1-e^{2}\right)}\left(\frac{a}{r_{a}}\right)^{3 / 2}
\end{gathered}
$$

Since Mercury has a total of 415.2 orbital cycles per century, the total discrepancy in the perihelion angle is given by

$$
\begin{gathered}
\Delta \phi=\frac{8 \pi G M_{s}}{c^{2} a\left(1-e^{2}\right)}\left(\frac{a}{r_{a}}\right)^{3 / 2}\left(\frac{360 \times 60 \times 60}{2 \pi}\right) 415.2= \\
43.2923 \text { arc seconds } / \text { century }
\end{gathered}
$$

Although Newton's theory had not been able to account for this, Einstein using General Relativity was able to calculate it [29] as

$$
\begin{gathered}
\Delta \phi=24 \pi^{3} \frac{a^{2}}{T^{2} c^{2}\left(1-e^{2}\right)}\left(\frac{360 \times 60 \times 60}{2 \pi}\right) 415.2= \\
42.9824 \text { arc seconds } / \text { century }
\end{gathered}
$$

According to astronomic observations the discrepancy angle is $45 \pm 5$ arc seconds/century.

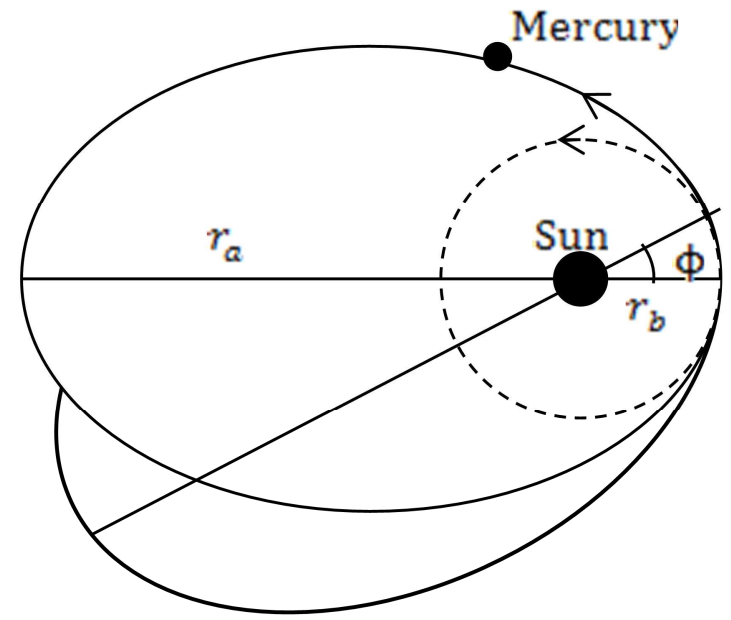

Figure 5. Schematic of Precession of the Perihelion of Mercury.

\subsection{Deflection of Light by the Sun}

Figure 6 shows a schematic of the bending of light caused by the gravitational acceleration of the sun. The total acceleration time dilation per unit of time at any point on the sun's surface, based on equation (33), is given by

$$
\delta t=2\left(z_{\text {sun }}^{2}-z_{\text {light }}^{2}\right)=2 z_{\text {sun }}^{2}=2 \frac{G M_{S}}{c^{2} R_{S}}
$$

Because light has no mass, $z_{\text {light }}^{2}=0$. The factor 2 in the above relation accounts for the fact that time dilates both as it approaches and as it leaves the sun's neighborhood. The angle $\theta$ is calculated by dividing the length of its chord by the radius, $R_{S}$. The length of the chord, $\Delta L$, associated with the time dilation, $\delta t$, is given by

$$
\Delta L=(c \delta t) \Delta t=(c \delta t) \frac{2 R_{s}}{c}
$$

Considering the fact that $\delta t$ is dimensionless, the term $(c \delta t)$ in the above equation represents the velocity, which is multiplied by the time, $\Delta t=\frac{2 R_{S}}{c}$, to give the length of the chord. Therefore based on equations (51) and (52) we get

$$
\begin{gathered}
\theta=\frac{\Delta L}{R_{S}}=\frac{(c \delta t) \frac{2 R_{S}}{c}}{R_{S}}=2 \delta t= \\
4 \frac{G M_{s}}{c^{2} R_{s}}
\end{gathered}
$$

Substitutions of the given data into the above equation yield

$$
\theta=4 \frac{G M_{S}}{c^{2} R_{S}}=4 \frac{\left(6.67408 \times 10^{-11}\right)\left(1.98855 \times 10^{30}\right)}{\left(2.99792458 \times 10^{8}\right)^{2}\left(6.65700 \times 10^{8}\right)} \frac{360 \times 60 \times 60}{2 \pi}=
$$

1.75126 arc seconds

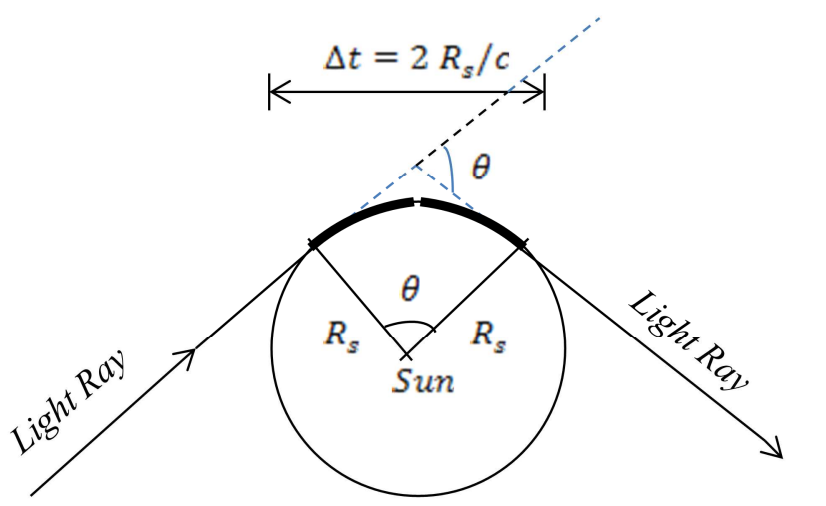

Figure 6. Schematic of Bending of Light Rays by the Sun.

\subsection{Gravitational Redshift}

We will show the effect of gravitational redshift by predicting the time dilation due to acceleration for a Geographic Positioning System Satellite [30, 31]. Based on the equivalence principle, we consider the spatial acceleration of the stretching-time to be equivalent to the acceleration due to gravity and will use the following data which are used in the current GPS operation

Speed of light $=2.998 \times 10^{8} \mathrm{~m} / \mathrm{s}$

Gravitational Constant $=G$

$=6.67408 \times 10^{-11} \mathrm{~m}^{3} \mathrm{~kg}^{-1} \mathrm{~s}^{-2}$,

Earth's Mass $=M_{e}=5.974 \times 10^{24} \mathrm{~kg}$

Earth's Radius $R_{e}=6.357 \times 10^{6} \mathrm{~m}$

Satellite's Height $=h=21.184 \times 10^{6} \mathrm{~m}$

$\lambda=10^{9} \times 24 \times 3600$ ns per 24 hours

Again based on equation (33), the time dilation due to earth's gravity between the earth and the Satellite is given by

$$
\delta t=z_{e}^{2}-z_{s}^{2}=\frac{G M_{e}}{c^{2} R_{e}}-\frac{G M_{e}}{c^{2} R_{s}}
$$

But

$$
R_{s}=R_{e}+h
$$

Substituting for $R_{S}$ from the above relation back into equation (55) yields the time dilation as 


$$
\delta t=z_{e}^{2}-z_{s}^{2}=\frac{G M_{e}}{c^{2}}\left(\frac{1}{R_{e}}-\frac{1}{R_{e}+h}\right)
$$

Substitutions for the different parameters from the given data into the above equation yield

$$
\delta t=5.30672 \times 10^{-10} \mathrm{~s} / \mathrm{s}
$$

For a 24 hour time span the above relation yields

$$
\delta t=\lambda \times\left(5.30672 \times 10^{-10} s / s\right)=45850 n s / d a y
$$

Using Schwarzschild's solution [32] of the Einstein equation, as shown below, yields essentially the same answer as

$$
\delta t=\lambda \times\left(\sqrt{1-\frac{2 G M_{e}}{c^{2} R_{e}}}-\sqrt{1-\frac{2 G M_{e}}{c^{2} R_{s}}}\right)=45850 n s / \text { day }
$$

\subsection{Consistency with Observational Data}

The above three tests validate the proposed model and demonstrate that the redshift, $z$, is an intrinsic property of time, that is, it is not a consequence of expansion of spacetime; it is its cause. At the present time the universe expands because the current of time flows with the cosmological redshift, $\mathrm{z}$, as its temporal velocity and

$$
\frac{d R\left(t_{0}\right)}{\mathrm{d} t}=c \frac{\mathrm{d} T_{S}\left(t_{0}\right)}{\mathrm{dt}}=c \mathrm{Z}
$$

as its spatial velocity, where $R\left(t_{0}\right)$ represents the presenttime value of the distance between the earth and the point source. Because the redshift, $\mathrm{z}$, acts as an amplification on the speed of light, the spatial velocity of stretching-time, $\frac{d R\left(t_{0}\right)}{\mathrm{d} t}$, for $\mathrm{z}>1$, exceeds the speed of light. This allows faster than light speed communication in time domain as it happens between entangled pairs of particles in quantum state. That is how temporal entanglement can happen.

To find the present-time value of the spatial distance between the earth and a point source, we multiply the present-time value of the stretching-time's spatial velocity, as given by equation (61), by the present-time value of the stretching-time, $T_{s}\left(t_{0}\right)$, as defined by equation (20). Thus, the spatial distance is given by

$$
R\left(t_{0}\right)=2 c \frac{\mathrm{d} T_{s}\left(t_{0}\right)}{\mathrm{dt}} T_{s}\left(t_{0}\right)=2 c z T_{s}\left(t_{0}\right)
$$

The factor 2 in the above relation, as noted in section 2, accounts for the fact that time flows forward both toward the earth and toward the point source simultaneously. Consequently the spatial length must be multiplied by the factor 2. Also to evaluate $T_{s}\left(t_{0}\right)$ using equation (20), due to time dilation effect as specified in equation (38), first the term $z$ in equations (17), (18), and (19) is replaced by $(z+\delta z)$. This substitution yields:

$$
\begin{gathered}
\mathrm{n}_{e}=\frac{((z+\delta z)+1) c t_{0}}{1.0632156 \times 10^{-3}}+\frac{1}{2} \\
\Delta \sigma_{i}=n_{e}-\frac{i}{13.8 m} \Delta \mathrm{n}=\left[\frac{((z+\delta z)+1) c t_{0}}{1.0632156 \times 10^{-3}}+\frac{1}{2}\right]- \\
\frac{i}{13.8 m} \frac{c t_{0}(z+\delta z)}{1.0632156 \times 10^{-3}}
\end{gathered}
$$

Using the above values for $\mathrm{n}_{e}$ and $\Delta \sigma_{i}$ in equation (20), then through equation (62) one obtains the value of the spatial distance as

$$
\begin{gathered}
R\left(t_{0}\right)=2 \text { c z } T_{s}\left(t_{0}\right)= \\
2 c z \sum_{n=\Delta \sigma_{i}}^{\text {ne }} \frac{2}{(2 n-1)} t_{o}, \quad 0 \leq i \leq m
\end{gathered}
$$

Clearly one only needs the age of the universe, $t_{0}$, and the redshift, $z$, to calculate the distance to any galaxy or star. To compare the spatial distances with the observational data, the distance, $R\left(t_{0}\right)$, as defined in equation (65), is converted to distance modulus via the following relation

$$
\mu=-5+5\left(\log _{10} \frac{R\left(t_{0}\right) \times G y}{\text { Parsac }}\right)
$$

where $\mu$ represents the distance modulus and Gy stands for giga year $\left(3.15576 \times 10^{16}\right.$ seconds $)$. The above equation is plotted in Figures 7 and 8 for the values of redshift, $z$, varying from 0 to 12 . To check how well the curve in these figures represents the reality, the following three sets of observational data are also plotted in these figures.

1. A set of $557 \mathrm{SNe}$ data with redshifts from a low of $\mathrm{z}=0.0152$ to a maximum of $\mathrm{z}=1.4$, as reported in 2010 in the Union2 Compilation [33]. In Figures 8 and 9 these data points are shown in Blue.

2. A set of 394 extragalactic distances to 349 galaxies at cosmological redshifts significantly higher than the Union2 Compilation with redshifts from a low of $\mathrm{z}=0.133$ to a maximum of $\mathrm{z}=6.6$, as reported in 2008 by Mador and Steer [34]. In Figures 7 and 8 these data points are shown in red.

3. A set of redshift data, as listed in Table 1, for the four spectroscopically confirmed galaxies. As only the redshift values are given, the corresponding distances for these four galaxies are evaluated based on equation (20). As such they have to match the curve perfectly. Their inclusion here serves to check the accuracy of the calculations. As the following two figures show, the analytical curve matches the observational data remarkably well

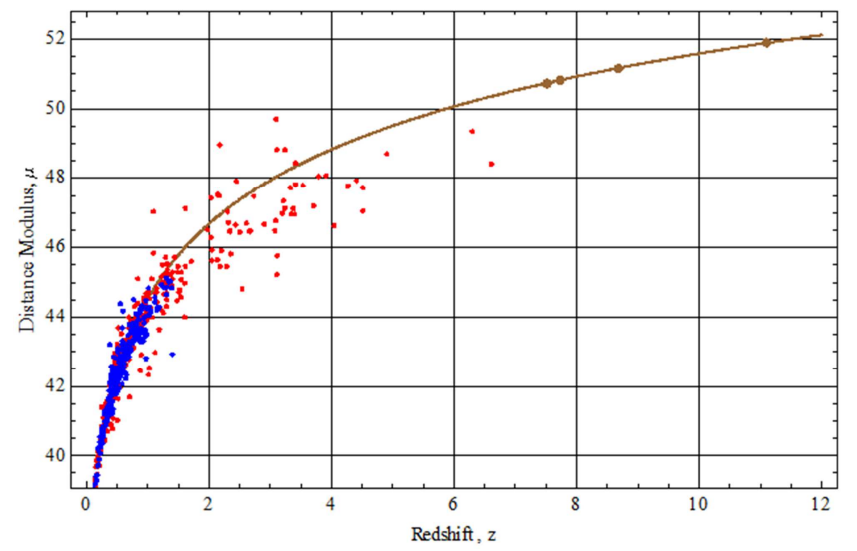

Figure 7. Comparison of the Analytical curve with the Observational Data. 


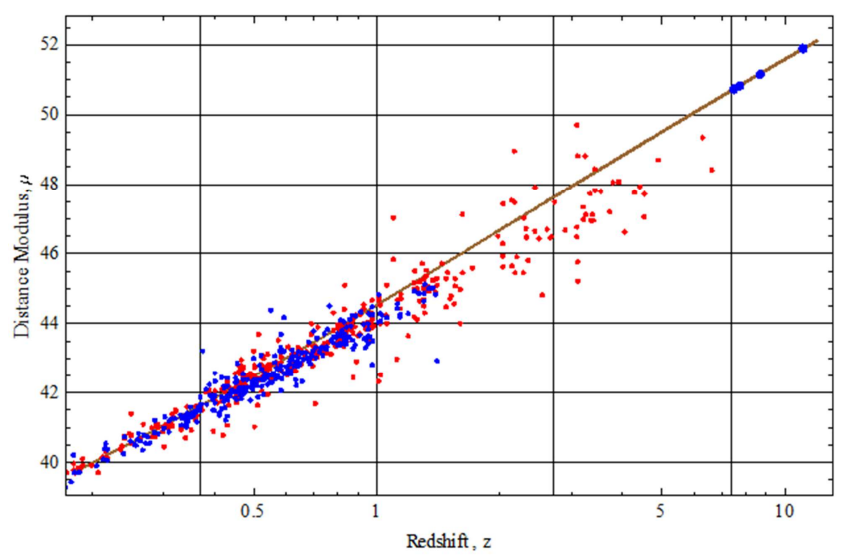

Figure 8. Comparison of the Analytical Curve with the Observational Data.

\section{Primordial Energy, Hubble Constant and Dark Energy}

So far all the formulations and results, from the beginning of section 2 through section 5, are based on classical mechanics. In the following we will see how the discrete process involved in the formulation of the stretching-time leads to a quantum mechanical approach to formulation of the primordial energy. Treating time as an independent variable, un-entangled with space-time, as shown in previous sections, revealed the discrete character of stretching-time. This discrete character suggests that the flow of time waves can be considered as streams of mass-less particles (energy in motion). This wave-particle treatment of time waves is consistent with the complementarity principle in quantum mechanics. We will use this discrete property to find the agent driving time forward. We will also use it to analytically estimate the Hubble constant and identify both the source and the quantity of what is called dark energy.

\subsection{The Driving Agent Behind Time}

Based on the assumed cosmological age of $13.8 \mathrm{~Gy}$, and equations (13) and (15) to (17), it can be shown that

$$
\begin{aligned}
& n_{o}=\frac{c t_{0}}{1.0632156 \times 10^{-3}}+\frac{1}{2}=1.22795 \times 10^{29} \\
& \mathrm{n}_{e}=\frac{\left(\frac{\tau_{\text {then }}}{2.72548}\right) c t_{0}}{1.0632156 \times 10^{-3}}+\frac{1}{2}=6.38338 \times 10^{60}
\end{aligned}
$$

where $\tau_{\text {then }}=1.41681 \times 10^{32} \mathrm{~K}$ is the Plank temperature. The Plank temperature is considered to be the temperature at the big bang. Clearly the number $n_{o}$ is a function of $t_{0}$, the assumed age of the universe. But the number $\mathrm{n}_{e}$, besides being a function of $t_{0}$, is also a function of the initial temperature, $\tau_{\text {then }}$, of the universe. The energy of a time wave of frequency $f_{n}$, according to the Plank-Einstein relation [35] and equation (8), is a constant defined by

$$
E_{n}=h f_{n}=\frac{\mathrm{h}}{p_{n}}=\frac{h}{t_{0}} \frac{(2 n-1)}{2}
$$

where $h$ is the Plank constant. Since the Plank temperature is a constant and the present-time age of the universe is assumed to be $13.8 \mathrm{~Gy}$, the numbers $n_{o}$ and $\mathrm{n}_{e}$, as given by equations (67) and (68), are also constants. Therefore the present-time value of the energy of the stretching-time, for a universe that has been assumed to have been at Plank temperature at the big bang, is given by

$$
\begin{gathered}
E=2 \sum_{n=n_{o}}^{n_{e}} h f_{n}=2 \sum_{n=n_{o}}^{n_{e}} \frac{\mathrm{h}}{p_{n}}= \\
2\left(\frac{\mathrm{h}}{t_{0}} \sum_{n=n_{o}}^{n_{e}} \frac{(2 n-1)}{2}\right)=6.19974 \times 10^{70} \text { joules }
\end{gathered}
$$

Factor 2 in the above relation, as noted in Figure 1, accounts for the fact that the stretching-time stretches both toward the left and the right. Time emerges as the energy is released. This primordial energy is released in quanta with frequencies ranging from $n_{o}$ to $n_{e}$ cycles/second. The stretching-time, which is the sum of the successive periods of these frequencies, starts its increase with the period corresponding to the frequency $n_{e}$ cycles/secon . As stretching-time starts to increase, the temperature begins to reduce. Considering the following relation

$$
E\left(t_{i}\right)=2 \frac{\mathrm{h}}{t_{0}} \sum_{n=\Delta \sigma_{i}}^{n_{e}} \frac{(2 n-1)}{2}, 0 \leq i \leq m
$$

where, based on equations (15) and (19), $\Delta \sigma_{i}$ is given by

$$
\begin{gathered}
\Delta \sigma_{i}=n_{e}-\frac{i}{13.8 m} \Delta \mathrm{n}= \\
{\left[\frac{\left(\frac{\tau_{\text {then }}}{2.72548}\right) c t_{0}}{1.0632156 \times 10^{-3}}+\frac{1}{2}\right]-\frac{i}{13.8 m} \frac{c t_{0}\left(\frac{\tau_{\text {then }}}{2.72548}-1\right)}{1.0632156 \times 10^{-3}},}
\end{gathered}
$$

one can see that there is an exact one to one correspondence between the terms in equation (71) and the terms in equation (20). This fact suggests that the driving agent that gives rise to the increase of the stretching-time, $\mathrm{T}_{\mathrm{s}}\left(t_{i}\right)$, as defined in equation (20), is the primordial energy that is released at the big bang. The released energy supplies the energy for the creation and propagation of time waves (or time particles). In fact the stretching-time represents the flow of this energy that has been propagating since the big bang. Thus this is the energy that by propagating the time waves gives rise to the accelerating expansion of space. As the energy drives the time waves forward the temperature drops and the stretchingtime increases. It is this increase in the stretching-time that, when multiplied by the spatial speed of time, appears as expansion of space.

The foregoing results are developed based on the stretching-time increasing both toward us and away from us. Physically the energy given in equation (70) spreads uniformly in all directions, driving the time waves forward. Therefore, at the present time, the trajectories of the stretching-time are all along great circles of the sphere of time. The spheres of time are nested inside each other. The diameter of each sphere is equal to the sum of the wave lengths divided by $N \pi$ where $N$ is the number of waves included in the summation. The time-rod in Figure 1, at the present time, represents the diameter of the largest sphere of time. The sphere of time has the cosmological age of the 
universe as its radius but its surface has no boundary.

The equivalent mass of the energy given by equation (70) is given by

$$
M=\frac{E}{c^{2}}=\frac{6.19974 \times 10^{70}}{c^{2}}=6.89815 \times 10^{53} \mathrm{~kg}
$$

This is the mass associated with the energy of time. Also throughout the emergence of time, from the big bang to the present, the equivalent total mass, $M_{\text {Total }}$, of all matter in our universe, remains constant. Therefore, since at the present time the redshift for earth is zero, based on equation (33), it is seen that the total mass of all matter in our universe, including the equivalent mass of all radiations and including the equivalent mass, as calculated in equation (73), is given by

$$
M_{\text {Total }}=\frac{c^{3} t_{p} z_{p}^{2}}{G}=\frac{c^{3} t_{p}\left(\frac{\tau_{p}}{2.72548}-1\right)^{2}}{G}=5.88151 \times 10^{55} \mathrm{~kg}
$$

Where $t_{p}$ and $\tau_{p}$ are the Plank time and the Plank temperature respectively and $z_{p}$ is defined by

$$
z_{p}=\frac{\tau_{p}}{\tau_{\text {now }}}-1=\frac{1.41681 \times 10^{32}}{2.72548}-1=5.19838 \times 10^{31}
$$

Thus the total energy of our universe is given by

$$
\begin{aligned}
E_{\text {Total }}= & c^{2} M_{\text {Total }}=\frac{c^{5} t_{p} z_{p}^{2}}{G}=\frac{c^{5} t_{p}\left(\frac{\tau_{p}}{2.72548}-1\right)^{2}}{G}= \\
& 5.28604 \times 10^{72} \text { joules }
\end{aligned}
$$

The above results together with the one-to-one correspondence of the terms in equation (20) with the ones in equation (71) suggest that a mega merger/collisions of supermassive bodies, whose total mass is given by equation (74), as a possible source of the energy given by equation (70). This is similar to recently discovered event of collision of two neutron stars that sat up gravitational waves and also released a large amount of energy [36, 37]. The released energy at the big bang has been propagating as the stretchingtime or primordial gravitational waves. The origin of time in our universe can be considered to be during this merger/collision of the super massive bodies at the big bang. This merger/collision is assumed to have taken place during an interval of time equal to the Plank time, at the big bang. As such, the mega merger/collision of super-massive bodies at the big bang explains the cause of the energy release without encountering any singularity. At the present time all trajectories of the stretching-time lie on the surface of the sphere of time. As the sphere of time expands with the passage of cosmological time, the temporal distances among all points on the surface of the sphere of time increase. The increasing temporal distance once multiplied by the spatial speed of the stretching-time, $d R\left(t_{0}\right) / d t$, gives rise to the increasing spatial distance.

The events of recently detected gravitational waves due to merging of neutron stars are similar to that at the big bang but as sources of gravitational waves they are closer to us than the source of time waves from the big bang. We have used equation (65) together with the age of the universe and the reported measured redshifts to calculate the distances to these events. These calculated distances along with the corresponding observationally measured distances are presented in Table 4. The consistency of the calculated values with the measured ones provides further validation for the time model.

Table 4. Comparison of Observational and Calculated Distances.

\begin{tabular}{lllll}
\hline Event & Reference & Redshift $\boldsymbol{z}$ & $\begin{array}{l}\text { Observed } \\
\boldsymbol{R}\left(\boldsymbol{t}_{\mathbf{0}}\right) \boldsymbol{M p} \boldsymbol{c}\end{array}$ & $\begin{array}{l}\text { Calculated } \\
\boldsymbol{R}\left(\boldsymbol{t}_{\mathbf{0}}\right) \boldsymbol{M} \boldsymbol{\boldsymbol { p }} \boldsymbol{c}\end{array}$ \\
\hline GW151226 & {$[13]$} & $0.09_{-0.04}^{+0.03}$ & $440_{-180}^{+160}$ & $399.8_{-187.8}^{+151.1}$ \\
GW150914 & {$[14]$} & $0.09_{-0.04}^{+0.03}$ & $420_{-180}^{+160}$ & $399.8_{-187.8}^{+151.1}$ \\
LVT151012 & {$[15]$} & $0.20_{-0.09}^{+0.09}$ & $1000_{-500}^{+500}$ & $995.3_{-497.7}^{+567.7}$ \\
GW170104 & {$[16]$} & $0.18_{-0.07}^{+0.08}$ & $880_{-390}^{+450}$ & $878.7_{-379.1}^{+487.3}$ \\
GW170814 & {$[17]$} & $0.11_{-0.04}^{+0.03}$ & $540_{-210}^{+130}$ & $499.6_{-195.7}^{+156.9}$ \\
\hline
\end{tabular}

\subsection{The Hubble Constant and Dark Energy}

We have not used the Hubble constant in the foregoing developments. However, if one considers the energy, $E$, as given in equation (70), to be vacuum or dark energy, then one obtains the following energy and mass densities for the vacuum:

$$
\begin{aligned}
& u_{v a c}=\frac{E}{\frac{4 \pi}{3}\left(2 c t_{0}\right)^{3}}=8.3135 \times 10^{-10} \text { joules } / \mathrm{m}^{3} \\
& \rho_{v a c}=\frac{E}{\frac{4 \pi}{3}\left(2 c t_{0}\right)^{3} c^{2}}=9.25002 \times 10^{-27} \mathrm{~kg} / \mathrm{m}^{3}
\end{aligned}
$$

Using the above value for the mass density of the vacuum one obtains the present-time value of the Hubble constant as

$$
\begin{aligned}
& H_{0}=\sqrt{\frac{8 \pi}{3} G \rho_{v a c}}=2.27419 \times 10^{-18}= \\
& 70.1404 \mathrm{~km} \mathrm{~s}^{-1} \mathrm{Mpc}^{-1}
\end{aligned}
$$

The most recent published values for the Hubble constant, based on observational data, are:

$73.0 \pm 1.75 \mathrm{~km} \mathrm{~s}^{-1} \mathrm{Mpc}^{-1}$ [38], 67.6 $6_{-0.6}^{+0.7} \mathrm{~km} \mathrm{~s}^{-1} \mathrm{Mpc}^{-1}$ [39], and 71.9 $9_{-3.0}^{+2.4} \mathrm{~km} \mathrm{~s}^{-1} \mathrm{Mpc}^{-1}$ [40]. The average of these three values is $70.83_{-1.78}^{+1.62} \mathrm{~km} \mathrm{~s}^{-1} \mathrm{Mpc}^{-1}$, which is within $1 \%$ of the analytically calculated value in equation (79). In arriving at the value for the energy used in equations (77) and (78), except for the assumptions implicit in Wien's displacement law and the temperature-redshift relation, no assumptions have been made about the ingredients forming the universe or about the geometry of space-time. Also no consideration has been given to the existence of a cosmological constant, vacuum or dark energy.

The closeness of the value of $H_{0}$, as given by equation (79), to the average of the measured value of the Hubble constant, as cited above, suggests that what is referred to as vacuum or dark energy is the energy of the stretching-time waves that, possibly as the result of the merger/collision of super-massive bodies, was released at the big bang. The present-time value of this energy, the energy of the stretching-time waves, is given by equation (70). Also the net energy left over after the merger/collision, excluding the energy of the stretching-time waves, as given in equation 
(70), is given by

$$
\begin{aligned}
\Delta E=E_{\text {Total }}-E= & 5.28604 \times 10^{72}-6.19974 \times 10^{70}= \\
& 5.22404 \times 10^{72} \text { joules }
\end{aligned}
$$

This energy is distributed among galactic bodies and through its gravity plays a significant role in the behavior of galaxies. A part of this energy is what is called dark matter and a small part of this energy constitutes the mass of ordinary matter and radiation. The rest of this energy is reserved for the future increase of the stretching-time and further evolution of our universe.

\section{Remarks and Conclusions}

The time model offers an alternative to the standard and the inflationary models of cosmology. It yields the timehistory of the expansion of our universe from the big bang to the present. It involves no assumptions except the ones implicit in Wein's displacement law and in the temperatureredshift relation. It also does not require the existence of a cosmological constant. The enormous accelerations and velocities given in Table 3 are due to the fact that, as one goes back in time, the radius of the sphere of time becomes smaller and smaller because the frequencies are becoming larger, causing the curvature of the trajectory of the stretching-time to grow. The agreement of the time model's analytical curve with the published observational data is remarkably good. Besides, the time model has yielded a simple equation based on the kinematics of time. Further the time model shows that the gravitational time dilation is the sole cause of precession of Mercury, the bending of light about the sun, and the gravitational redshift. Also the time model has revealed both the source and the quantity of what is called dark energy. Further, the model shows that the time waves are driven by the energy emanating from the big bang and propagating in all directions. In addition, the occurrence of the event that gave rise to the emergence of our big bang did not have to have been a unique incident. As such the time model does not exclude the existence of time before the big bang and leaves open the possibility of the existence of other universes.

As implied by the Plank-Einstein relation, like other constants of nature, each time wave has an intrinsic amount of energy which is a constant. At the big bang the energy is released in quanta with distinct frequencies. The superposition of the periods of time waves causes the stretching-time to increase because, as the energy is released in quanta, the stretching-time increases by a "quantum leap" from one sphere of time to the next, while adding one more period to its summation. Each of the infinite numbers of points on the surface of the sphere of the present-time represents the same present-time.

As a consequence of isolating the role of time from spacetime, it is seen that the increase in the stretching-time is a discrete process. This discrete behavior of time appears to clear the way to combining quantum mechanics and gravity and to a quantum mechanical description of the cosmos.

\section{Acknowledgements}

All the numerical work and plots are done via Mathematica v.10.0, 4/3/2014.

\section{References}

[1] Hubble, E., "A Relation between Distance and Radial Velocity among Extra-Galactic Nebulae, 1929," Proceedings of the National Academy of Sciences of the United States of America, 15:3, 168-173 (1929). http://www.pnas.org/content/15/3/168.

[2] Perlmutter, S, 1998, "Supernovae, Dark Energy, and the Accelerating Universe: The Status of the Cosmological Parameters," Supernova Cosmology Project, Lawrence Berkeley National Laboratory, Berkeley, CA 94720, www.slac.stanford.edu/econf/C990809/docs/perlmutter.pdf

[3] Perlmutter, S. (2012), "Nobel Lecture: Measuring the Acceleration of the Cosmic Expansion. Using Supernovae," Review of Modern Physics, Vol. 84, pp. 1127-1149.

[4] Riess, A. G. (2012), "Nobel Lecture: My Path to the Accelerating Universe," Reviews of Modern Physics, Vol. 84, pp. 1165-1175.

[5] Schmidt, B. P. (2012), "Nobel Lecture: Accelerating Expansion of the Universe through Observation of Distant Supernovae," Reviews of Modern Physics, Vol. 84, pp. 11511163.

[6] Guth, A H, 1981 "The inflationary universe: A possible solution to the horizon and flatness problems," Phys. Rev. D $23,347-356$.

[7] Guth, AH, 2002, "Inflation and the New Era of HighPrecision Cosmology - MIT, Inflation and the New Era of High-Precision Cosmology - MIT.

[8] Guth, A H, 1981 "The inflationary universe: A possible solution to the horizon and flatness problems," Phys. Rev. D $23,347-356$.

[9] Zeldovich, Y. B., 1970, "Gravitational Instability: An Approximate Theory for Large Density Perturbations," Astron. \& Astrophys. 5, 84-89 (1970).

[10] Albrecht, A., Steinhardt, P. J., 1982, "Cosmology for Grand Unified Theories with Radiatively Induced Symmetry," Physical Review Letters, 481220 (1982).

[11] Linde, A., 1998, "A Toy Model for Open Inflation," Phys, Rev. D 59 (1999), ArXiv:hep-ph/9807493.

[12] Hawking, S. W., Turok Neil, 2011, "Open Inflation Without False Vacua," ArXiv:hep-th/9802030v1.

[13] Abbott et al., 2016, "GW151226: Observation of Gravitational Waves from a 22-Solar-Mass Binary Black Hole Coalescence," Phys. Rev. Lett. 116, 241103 (2016).

[14] Abbott et al., 2016, "Observation of Gravitational Waves from a Binary Black Hole Merger,” Phys. Rev. Lett. 116, 061102, (2016). 
[15] Abbott et al. 2016, "Binary Black Hole Mergers in the First Advanced LIGO Observing Run,” Phys. Rev. X 6, 041015 (2016).

[16] Abbott et al., 2017, "GW170104: Observation of a 50-SolarMass Binary Black Hole Coalescence at Redshift 0.2," Phys. Rev. Lett. 118, 221101 (2017).

[17] Abbott et al., 2017, "GW170814: A Three-Detector Observation of Gravitational Waves from a Binary Black Hole Coalescence," Phys. Rev. Lett. 119, 141101 (2017).

[18] Clough, R W, Penzien, J, 1975, "Dynamics of Structures," McGraw-Hill.

[19] Seto, WW, 1964, "Theory and Problems of Mechanical Vibrations," Schaum Publishing Co New York.

[20] Fixsen, D. J. (2009). "The Temperature of the Cosmic Microwave Background". The Astrophysical Journal. 707 (2): 916-920. arXiv:0911.1955 [astro-ph.CO] 10, Nov.

[21] NIST, CODATA VALUE: Wien Wavelength Displacement Law Constant. http://physics.nist.gov/cgibin/cuu/Value?bwien

[22] Wikipedia, Wien's displacement law - Wikipedia https://en.wikipedia.org/wiki/Wien\%27s_displacement_law

[23] Jarosik, N., et. al., "Seven-Year Wilkinson Microwave Anisotropy Probe Observations: Sky Maps, Systematic Errors and Basic Results,” Astrophys. J., Suppl. Ser. 192, 14, 2011.

[24] Oesch, P A, Brammer, G, Van Dokkum, P G, 2016, “A Remarkably Luminous Galaxy at $\mathrm{Z}=11.1$ Measured with Hubble Space Telescope Crism Spectroscopy," ArXiv: 1603.00461v1 [astro-ph.GA] 1, March 2016.

[25] Zitrin, A., Labbe, I., Belli, S., et al., 2015, "Lyman-Alpha Emission From A Luminous $\mathrm{z}=8.68$ Galaxy: Implications for Galaxies as Tracers of Cosmic Reionization," ArXiv:1507.02679v3 [astro-ph.GA] 13, Aug 2015 https://en.wikipedia.org/wiki/List_of_the_most_distant_astron omical_objects

[26] Oesch, P. A., Van Dokkum, P. G., Illingworth, G. D., 2015, “A Spectroscopic Redshift Measurement for a Luminous Lyman Break Galaxy at $\mathrm{z}=7.730$ Using Keck/Mosfire," ArXiv: 1502.05399 v2 [astro-ph.GA] 3, May 2015

[27] Finkelstein, S. L., Papovich, C., Dickinson, M., et al., 2013, "A Rapidly Star-forming Galaxy 700 Million Years After the Big Bang at $\mathrm{z}=7.51$, AarXiv: 1310.6031v1 [astro-ph.GA] 22, Oct 2013.

[28] Riley, F., Sturges, L. D., 1993, "Engineering Mechanics, Dynamics," John Wiley \& Sons.
[29] Vankov, Anatoli A, 2010, Einstein's Paper: "Explanation of the Perihelion Motion of Mercury from General Relativity Theory" http://adsabs.harvard.edu/abs/2010arXiv1008.1811V

[30] GPS, Global Positioning System Precise Positioning Service Performance Standard, February 2007, Department of Defence, USA, www.gps.gov/technical/ps/2007-PPSperformance-standard.pdf

[31] Wikipedia, Error analysis for the Global Positioning System Wikipedia https://en.wikipedia.org/wiki/Error_analysis_for_the_Global_ Positioning_System

[32] Wikipedia, Gravitational time dilation - Wikipedia https://en.wikipedia.org/wiki/Gravitational_time_dilation

[33] Amanullah, R., Lidman, C., Rubin, D., Aldering, G., et al., 2010, "Spectra and Hubble Space Telescope Light Curves of Six Types Ia Supernovae at $0.511<\mathrm{z}<1.12$ and the Union2 Compilation," The Astrophysical Journal, 716:712-738, data available at http://supernova.lbl.gov/Union/figur...n2_mu_vs_z.txt

[34] Mador, B. F., Steer, I. P., 2008, "NASA/IPAC Extragalactic Database Master List of Galaxy Distances," NED-4D, http://ned.ipac.caltech.edu/level5/NED4D/

[35] Berkeley Lecture 8.1, Representations and Wave functions, C/CS/Phys C191, Fall 2008, https://inst.eecs.berkeley.edu/ cs191/fa08/lectures/lecture8_fa 08.pdf

[36] Conover, E., This year's neutron star collision unlocks cosmic mysteries, Science New, Vol. 192, No. 11, December 23, 2017, p. 19.

[37] Shappee, B J, et al. Early spectra of the gravitational wave source GW170817: Evolution of a neutron star merger. Science. Published online October 16, 2017. doi: 10.1126/science.aaq0186

[38] Riess, A. G, Macri, L M, Hoffmann, S L, et al, 2016, "A 2.4\% Determination of the Local Value of the Hubble Constant," ArXiv:1604.01424v3 [astro-ph.CO] 9 Jun 2016.

[39] Grieb, J N, Sanchez, A G, Salazar-Albornoz, S, et al, 2016, "The Clustering of Galaxies in the Completed SDSS-III Baryon Oscillation Spectroscopic Survey ...," ArXiv:1607.03143v1 [astro-ph.CO] 11 July 2016.

[40] Bonvin, V, Courbin, S H, Suyu, P J, etal, 2017, "H0LiCOW V. New Cosmograil time delays of HE0435-1223: $\mathrm{H}_{0}$ to $3.8 \%$ precision from strong lensing in a flat CDM model," ArXiv:1607.01790v2 [astro-ph.CO] 25 Jan 2017. 